\section{ULTRASONOGRAPHY: coping with changes and setting new directions after being listed in SCIE}

JEONG-SIK YU

Editor-in-Chief ULTRASONOGRAPHY

After being notified that Ultrasonography was listed in the Science Citation Index Expanded (SCIE) last September, we received many congratulations from various parties [1]. Aside from the congratulations to myself and the editorial staff, I believe that the Korean Society of Ultrasound in Medicine (KSUM) itself should be congratulated for the objective confirmation of its status through this achievement, which resulted from our society's tireless efforts to develop the role of ultrasound in medicine. Now, as the editor-in-chief of Ultrasonography, it is time to think about how to embark in new directions to cope with the changes that we expect after being listed in SCIE and how to achieve the goals that fall within our scope.

The number of submissions has tripled in the last few months since we were listed in SCIE compared to the previous year. The increasing number of submissions has placed a heavy burden on editors and reviewers. Currently, when a paper is submitted, the section editor first determines whether the content of the paper fits the scope of our journal, decides whether to conduct peer review for publication, and selects two or more peer reviewers. The current global trend is for fewer peer reviewers to be willing to devote time to reviewing submissions, both because the number of papers to review is growing and because reviewers are overworked. Of course, now that Ultrasonography is listed in SCIE, I would like to hope that reviewers will be more likely to agree to write a review for our journal when requested. In reality, however, the reviewer pool should be increased globally, and some compensation may be needed for reviewers. I also think that it will be helpful to find more suitable reviewers through internet-based services such as Publons (https://publons.com). In the future, if the number of submissions increases to the point that it becomes difficult to handle with the current system, each section editor will need to limit the number of articles to be sent for peer review by evaluating the value of the articles in advance. This will be accompanied by appropriate compensation for the section editor.

If we are faced with a major influx of submissions, our first option is to consider ways to publish the same number of issues and articles by applying stricter selection criteria to limit the number of accepted papers. However, in order to include publications on technological

\section{Jeong-Sik Yu, MD}

Department of Radiology, Gangnam Severance Hospital, Yonsei University College of Medicine, 211 Eonju-ro, Gangnam-gu, Seoul 06273, Korea Tel. +82-2-2019-3510, Fax.+82-2-3462-5472, E-mail: yjsrad97@yuhs.ac

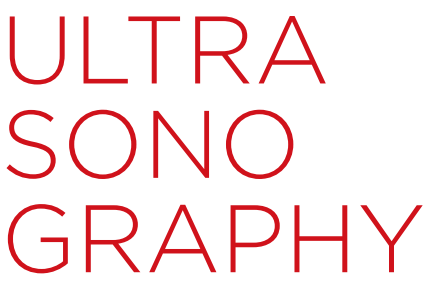

\section{EDITORIAL}

https://doi.org/10.14366/usg.19080 pISSN: 2288-5919 • elSSN: 2288-5943 Ultrasonography 2020;39:1-2

Received: November 27, 2019

Revised: November 27, 2019

Accepted: November 30, 2019

This is an Open Access article distributed under the terms of the Creative Commons Attribution NonCommercial License (http://creativecommons.org/ licenses/by-nc/4.0/) which permits unrestricted noncommercial use, distribution, and reproduction in any medium, provided the original work is properly cited.

Copyright @ 2020 Korean Society of Ultrasound in Medicine (KSUM)

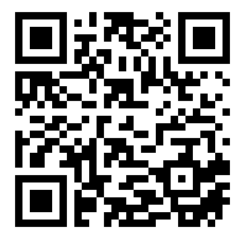

How to cite this article:

Yu JS. ULTRASONOGRAPHY: coping with changes and setting new directions after being listed in SCIE. Ultrasonography. 2020 Jan;39(1):1-2. 
advances and accumulated clinical experiences, it may be necessary to increase the number of articles published in each issue or to increase the number of issues. In the short term, I think that the system we have been using in which electronic publications are made available ahead of print has significantly contributed to the early exposure received by our articles. However, a problem is that the interval until the official publication has become excessively long after electronic publications are released. Another option would be to stop printing hard copies and to fully convert Ultrasonography into an e-journal.

As submissions become more numerous, the costs of review, editing, and publication will increase. Until now, all of these processes have been primarily supported by KSUM. However, KSUM funding alone will not be able to meet this increased financial burden. At some point, I expect the day to come for authors to pay processing fees for articles and for the costs of open access, but it is difficult to predict when Ultrasonography will become an economically independent journal.

As increasingly many original articles are submitted and published, the proportion of review articles will decrease. It was previously possible to increase the citation score and impact factor (IF) of our journal by actively recruiting domestic and international scholars to write reviews. Increasing the publication of original articles with relatively few citations may lead to a decrease in citation metrics. In the long run, the ideal solution would be to increase the citations of original articles by improving the quality of published manuscripts. However, it is inherent to the nature of medical ultrasonography that citations of original articles are limited. For example, the overall citation rate of Ultrasound in Medicine and Biology, the official journal of the World Federation for Ultrasound in Medicine and Biology (WFUMB), which favors the publication of original articles, is lower than ours. Unless we intend to accept a reduction in IF, we need to attract more high-impact review articles, even if they are relatively few, to maintain or increase the citation rate in the future. As mentioned previously [2], we plan to take steps to increase our IF in the short term by attracting up-to-date guidelines on ultrasonography and by publishing artificial intelligencerelated content, as well as other review articles.

To cope with the changing environment without merely settling for the status of an SCIE-listed journal, it is still necessary to solve the problem of human and material costs. Ultimately, as our goal is to become a globally leading medical ultrasonography journal, we need effective short- and longterm strategies for increasing the quantity and improving the quality of the content published in Ultrasonography. Starting this year, we will hold an annual editorial workshop at the KSUM conference (for which the 2020 website is open, at https://2020.ksum.or.kr) to listen to the opinions of experts and to help establish concrete strategies through active discussions.

ORCID: Jeong-Sik Yu: https://orcid.org/0000-0002-8171-5838

\section{References}

1. YU JS. ULTRASONOGRAPHY is now in SCIE. Ultrasonography 2019;38:277.

2. YU JS. ULTRASONOGRAPHY: how to raise the impact factor. Ultrasonography 2017;36:1-2. 